\title{
Preventive chemotherapy to control soil-transmitted helminthiasis averted more than 500000 DALYs in 2015
}

\author{
A. Montresor ${ }^{a, *}$, W. Trouleau ${ }^{b}$, D. Mupfasoni ${ }^{a}$, M. Bangert ${ }^{a}$, S. A. Joseph ${ }^{a}$, A. Mikhailov ${ }^{a}$ and C. Fitzpatrick ${ }^{a}$ \\ ${ }^{a}$ Department of Control of Neglected Tropical Diseases, World Health Organization, Geneva; ${ }^{b}$ School of Computer and Communication \\ Sciences, EPFL, Lausanne, Switzerland \\ *Corresponding author: E-mail: montresora@who.int
}

Received 28 September 2017; revised 22 November 2017; editorial decision 13 December 2017; accepted 19 December 2017

\begin{abstract}
Background: Preventive chemotherapy (PC), the large-scale administration of anthelminthics, is recommended by the World Health Organization (WHO) for the control of soil-transmitted helminthiasis (STH). Since 2010, donated anthelminthics for STH have boosted the implementation of PC programmes in children, achieving global coverage of more than 60\% in 2015. The WHO Global Health Estimates attribute an annual loss of over 3.3 million disability-adjusted life-years (DALYs) to STH. The aim of this study is to estimate the impact of PC programmes on child morbidity.
\end{abstract}

Method: We used data from the WHO Global Health Estimates, national coverage data on PC and the results of an evaluation of the impact of PC in 17 countries on morbidity previously conducted by our group.

Results: We estimated that the implementation of PC averted in 2015 over $44 \%$ of the DALYs that would have been caused in children by STH without the control intervention. A reduction in morbidity of over $75 \%$ is expected, if the global target is reached in 2020. If the programme is subsequently maintained, morbidity from STH will be almost totally removed by 2025 .

Conclusions: In endemic areas, preventive chemotherapy provides a significant health benefit. We consider this estimation potentially useful to evaluate the cost utility of the investment made by several endemic countries on PC to control STH.

Keywords: A.lumbricoides, T. trichiura, hookworms, soil transmitted helminth, morbidity, DALYs

\section{Introduction}

Over 1 billion people are infected with soil-transmitted helminths (STH), a group of intestinal parasites that are intensively transmitted in tropical and subtropical areas, especially where water supply and sanitation infrastructure are inadequate. ${ }^{1}$ The STH group includes four species of worms: Ascaris lumbricoides, Trichuris trichiura, and the hookworms Necator americanus and Ancylostoma duodenale.

The morbidity caused by STH results from a disturbance of the normal nutritional processes; the exact mechanism by which this occurs varies by species. ${ }^{2}$ STH morbidity is proportional to the number of worms infecting the host. For A. lumbricoides and $T$. trichiura, only infections of moderate to heavy intensity are considered to cause damage to health, while hookworm infections of all intensities are linked to morbidity. ${ }^{3}$

The WHO Global Health Estimates ${ }^{4}$ measure the annual health burden of all human diseases and those attributed to STH, globally, for all ages, a loss in 2010 of 3.394 million disabilityadjusted life-years (DALYS). WHO estimates attribute morbidity due to A. lumbricoides and T. trichiura only if these infections are of moderate and heavy intensity; hookworm infections of any intensity are considered to cause morbidity.

Preventive chemotherapy $(\mathrm{PC})$ is the strategy recommended by WHO to control morbidity attributed to STH and other selected neglected tropical diseases (NTD). ${ }^{3}$ Pre-school children (pre-SAC), school-age children (SAC) and women of reproductive age (WRA) are considered particularly vulnerable to STH infections, and WHO recommends the periodic treatment of these groups at risk in countries endemic for STH. The recommended frequency of the PC intervention is once or twice per year according the STH prevalence in the population at baseline.

Global coverage of this intervention has progressively increased in children from less than $30 \%$ in 2010 to $30 \%$ in $2011,35 \%$ in 2012, 40\% in 2013, 43\% in 2014 to over 58\% in 2015 and is expected to reach the target set by WHO of $75 \%$ by $2020^{5}$

(c) The Author(s) 2018. Published by Oxford University Press on behalf of Royal Society of Tropical Medicine and Hygiene. All rights reserved. For permissions, please e-mail: journals.permissions@oup.com. 


\section{A. Montresor et al.}

Marocco et al. ${ }^{6}$ measured the effect of PC on the morbidity of each species of STH in 17 control programmes for which parasitological baseline and follow up data at different intervals were available. In that study we considered the morbidity associated with infections due to A. lumbricoides and T. trichiura of moderate and heavy intensity (if only infections of light intensity remained in a population group, we considered the morbidity for those parasites as completely eliminated); for hookworm we considered any infection to cause morbidity, in line with the WHO Global Health Estimates. ${ }^{4}$

The study showed that the efficacy of PC drugs varies depending on the different STH species. However, after 1 year of treatment the number of infections of moderate and heavy intensity for each STH species were reduced by over 75\%. Over time, and with multiple rounds of PC according to the WHO-recommended schedule, $^{7}$ the reduction approaches $100 \%$. This study makes it possible to predict reductions in STH-related morbidity over time.

WHO has managed the donation of anthelminthics to control STH since 2011: every year, data on PC coverage in each endemic country are collected and validated before new amounts of anthelminthics are donated. These data are updated annually ${ }^{8}$ and published online in the WHO NTD databank. ${ }^{9,10}$

The objectives of this study are to estimate the number of DALYs that have been averted in children in 2015 by PC implemented during 2010-2015 and to estimate the number of DALYs that will be averted in 2020 if PC coverage is expanded to reach the global target of $75 \%$.

\section{Methods}

This study aims to estimate the number of DALYs averted in 2015 by extrapolating the results measured by Marocco et al. ${ }^{6}$ in 17 countries (where classes of intensity of infection were measured at baseline and follow up) to all the other countries receiving PC for STH in which an estimation of the baseline morbidity is available ${ }^{4}$ but follow up data on intensity of infection are not.

For this purpose, in each endemic country, we applied to the DALYs attribute to STH in 2010, a reduction corresponding to the length of implementation of PC as calculated by Marocco et al. ${ }^{6}$ The reduction was applied proportionally to the population receiving PC (i.e. if only half of the SAC population in a country has received 5 consecutive years of $P C$ and the rest nothing, we applied the reduction in DALYs corresponding to 5 years, only to the half of the population treated and we assumed that in the rest the population, the DALYS caused by STH remained unchanged).

To perform this calculation, we needed, for each STH endemic country:

- the morbidity (in DALYs) attributed to STH in 2010;

- the PC coverage data 2010-2015.

\section{STH morbidity in the absence of PC interventions}

A number of studies estimating the burden of STH in DALYS have been conducted in recent years. ${ }^{11,12}$ We selected the DALY estimation in WHO Global Health Estimate ${ }^{4}$ as the basis for our study because these data are freely available online, and provide the estimates by country, age group, and by each of the different STH parasites.

For each STH endemic country we extracted from the WHO Global Health Estimates ${ }^{4}$ the number of DALYs lost to $A$. lumbricoides, T. trichiura and hookworm infections in the absence of large-scale PC interventions (i.e., in 2010). The data were summarized globally, by STH species and by at-risk groups (Table 1).

\section{PC coverage data}

The WHO NTD databank ${ }^{10}$ collects coverage data from all countries that implement PC, and that request donations of albendazole or mebendazole.

We are confident that these coverage data are of high quality because after submission from the manager of the national control programme they are scrutinized and, in case of doubt, the manager is requested to explain unexpected high or low coverage, discrepancies between the amount of number of donated medicines and the number distributed, and discrepancies between the reported number of treatments and the amount of remaining medicine. This exercise of data verification can take several months from the date of data submission (normally in August of every year) and publication (normally in December of every year).

From the NTD databank ${ }^{10}$ we extracted for each endemic country:

- the number of pre-SAC in need of treatment, each year for 2010-2015.

- PC coverage in pre-SAC, each year for 2010-2015;

- the number of SAC in need of treatment, each year for 2010-2015;

- PC coverage in SAC, each year for 2010-2015.

From the PC national coverage data for 2010-2015 we calculated in each country the percentage of the population of children (pre-SAC and SAC separately) that were treated for 1, 2, 3, 4,5 or 6 years with the following method.

In the case of monotonically increasing $P C$ coverage in a country, the PC national coverage in 2010 corresponds to the percentage of children treated consecutively for 6 years (from 2010 to 2015). The difference between the PC national coverage in 2011 and 2010 corresponds to the percentage of children treated consecutively for 5 years (from 2011 to 2015), and so on. In Figure 1(a), we present the case of Senegal, where the PC national coverage in SAC started at $14 \%$ in 2010 and reached $70 \%$ in 2015 . In this example we assumed that:

- $14 \%$ of SAC received 6 years of treatment;

- $10 \%$ of SAC received 5 years of treatment;

- $36 \%$ of SAC received 4 years of treatment;

- $3 \%$ of SAC received 3 years of treatment;

- $25 \%$ of SAC received 2 years of treatment;

- $6 \%$ of SAC received 1 year of treatment.

Using the percentages of children treated consecutively for each time length between 2010 and 2015 (as presented in Figure 1(a)), along with the curves described by Marocco et al., ${ }^{6}$ it was then possible to calculate the percentage of DALYS expected to be averted by PC.

In the case where expansion of PC coverage was not monotonically increasing (i.e. PC national coverage in the country decreased in one or more years), we were not able to directly apply the same methodology since our estimation on 
Table 1. Estimated number of disability-adjusted life years (DALYS) lost every year (without intervention) in high-risk groups and the number averted by preventive chemotherapy by 2015 and by 2020

\begin{tabular}{|c|c|c|c|}
\hline Category & $\begin{array}{l}\text { STH burden (2010): DALYs lost } \\
\text { for STH without intervention. } \\
\text { (thousands). }\end{array}$ & $\begin{array}{l}\text { DALYs averted in } 2015^{* *} \text { by } \\
\text { PC 2010-2015 (thousands). }\end{array}$ & $\begin{array}{l}\text { DALYs expected to be } \\
\text { averted in } 2020^{* *} \text { by PC } \\
2010-2020 \text { (thousands). }\end{array}$ \\
\hline STH (in children) & 1262 & $549(44 \%)$ & 909 (73\%) \\
\hline A. lumbricoides & 539 & $284(53 \%)$ & $408(76 \%)$ \\
\hline T. trichiura & 150 & $61(41 \%)$ & $108(72 \%)$ \\
\hline Hookworms & 571 & $204(36 \%)$ & $393(69 \%)$ \\
\hline \multicolumn{4}{|l|}{ By high- risk group } \\
\hline Pre-school children (all species) & 396 & $180(45 \%)$ & $295(74 \%)$ \\
\hline A. lumbricoides & 249 & $124(50 \%)$ & $190(76 \%)$ \\
\hline T. trichiura & 30 & $14(45 \%)$ & $24(76 \%)$ \\
\hline Hookworms & 116 & $41(35 \%)$ & $81(69 \%)$ \\
\hline School-age children (all species) & 862 & $368(43 \%)$ & $613(71 \%)$ \\
\hline A. lumbricoides & 287 & $158(55 \%)$ & $215(75 \%)$ \\
\hline T. trichiura & 121 & $48(40 \%)$ & $86(71 \%)$ \\
\hline Hookworms & 454 & $163(36 \%)$ & 312 (69\%) \\
\hline \multicolumn{4}{|l|}{ by region } \\
\hline AFR* (all species) $^{*}$ & 488.8 & $211.4(43 \%)$ & $353.2(72 \%)$ \\
\hline A. lumbricoides & 224.5 & $119.4(53 \%)$ & $172(76 \%)$ \\
\hline T. trichiura & 51.5 & $15.6(30 \%)$ & $35.6(69 \%)$ \\
\hline Hookworms & 212.8 & $76.3(36 \%)$ & $145.4(68 \%)$ \\
\hline Pre-school children & 178.8 & $88.0(49 \%)$ & $142.9(80 \%)$ \\
\hline School age children & 310.0 & $123.4(40 \%)$ & $210.2(68 \%)$ \\
\hline AMR* (all species) & 106.7 & $33.1(31 \%)$ & $69.8(65 \%)$ \\
\hline A. lumbricoides & 36.1 & $12.8(35 \%)$ & $24.6(68 \%)$ \\
\hline T. trichiura & 21.7 & $9.2(42 \%)$ & $16.1(74 \%)$ \\
\hline Hookworms & 48.9 & $10.9(22 \%)$ & $29.7(59 \%)$ \\
\hline Pre-school children & 27.9 & 7.7 (28\%) & $18.1(365 \%)$ \\
\hline School age children & 78.8 & $25.3(32 \%)$ & $51.6(65 \%)$ \\
\hline SEAR* (all species) & 381.7 & $220.6(58 \%)$ & $302.3(79 \%)$ \\
\hline A. lumbricoides & 172.1 & $109.6(64 \%)$ & 133.9 (78\%) \\
\hline T. trichiura & 27.2 & $18.1(67 \%)$ & $23.2(86 \%)$ \\
\hline Hookworms & 182.5 & $92.8(51 \%)$ & $145.1(80 \%)$ \\
\hline Pre-school children & 107.1 & $44.7(42 \%)$ & $72.5(68 \%)$ \\
\hline School age children & 274.6 & $175.8(64 \%)$ & $232.7(84 \%)$ \\
\hline EUR* (all species) & 3.5 & $1.1(31 \%)$ & $3.1(87 \%)$ \\
\hline A. lumbricoides & 3.5 & $1.1(31 \%)$ & $3.1(87 \%)$ \\
\hline T. trichiura & 0.018 & 0 & $0.011(60 \%)$ \\
\hline Hookworms & 0.002 & 0 & $0.001(50 \%)$ \\
\hline Pre-school children & 1.7 & $0.6(35 \%)$ & $1.7(100 \%)$ \\
\hline School age children & 1.9 & $0.4(21 \%)$ & $1.2(65 \%)$ \\
\hline EMR* (all species) $^{*}$ & 76.1 & $28.2(37 \%)$ & $54.9(72 \%)$ \\
\hline A. lumbricoides & 58.5 & $25.5(44 \%)$ & $44.6(77 \%)$ \\
\hline T. trichiura & 1.9 & $0.3(16 \%)$ & $1.1(64 \%)$ \\
\hline Hookworms & 15.7 & $2.4(15 \%)$ & $9.1(58 \%)$ \\
\hline pre-school children & 35.6 & $23.5(66 \%)$ & $30.7(86 \%)$ \\
\hline school age children & 40.5 & $4.7(12 \%)$ & $24(60 \%)$ \\
\hline WPR* (all species) & 205.1 & $55.2(27 \%)$ & $127(62 \%)$ \\
\hline A. lumbricoides & 44.2 & $15.3(35 \%)$ & $29.3(67 \%)$ \\
\hline T. trichiura & 49.2 & $18.4(37 \%)$ & $32.7(66 \%)$ \\
\hline Hookworms & 111.7 & $21.5(19 \%)$ & $64.9(57 \%)$ \\
\hline Pre-school children & 48.3 & $16.8(35 \%)$ & $31(64 \%)$ \\
\hline School age children & 156.8 & $38.3(24 \%)$ & $95.8(61 \%)$ \\
\hline
\end{tabular}

${ }^{*} \mathrm{AFR}=$ African Region; AMR = The Americas Region; EMR = Eastern Mediterranean Region; EUR = European region; SEAR = South East Asia Region; WPR = Western Pacific Region.

${ }^{* *}$ Compared with 2010 DALY. 

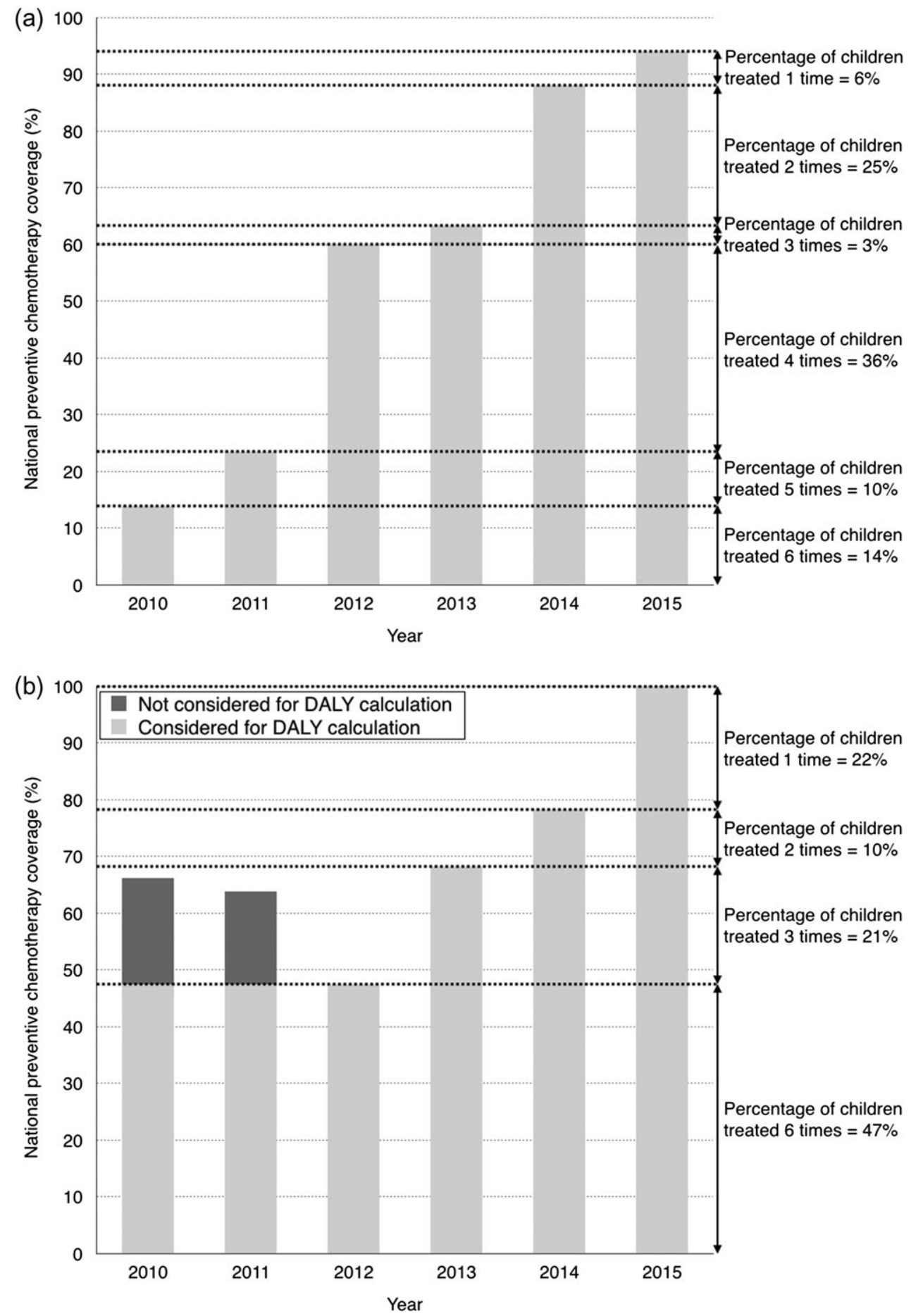

Figure 1. Example of calculation of the number of children treated one, two, three, four, five and 6 times from the annual coverage figures 2010 2015. (a) Coverage of school age children in Senegal (monotonical increase). (b) Coverage of SAC in Vietnam (non-monotonical increase).

morbidity reduction due to $\mathrm{PC}$ is calculated only for consecutive treatments. In this case, we decided to be conservative in measuring the DALY reduction, and the percentage of children with interrupted treatment was not taken into account in the estimation of the number of DALYs averted. An example of the methodology is presented in Figure 1 (b) for Vietnam, where in 2012 the national coverage of SAC was lower than in the previous 2 years. The percentage of children treated in 2010 and 2011 exceeded the percentage covered in 2012 and was not taken into account for the calculation of the number of DALYs averted.

For each endemic country we calculated the percentage of children (separately for pre-SAC and SAC) that were treated for $1,2,3,4,5$ and 6 years with PC in the period 2010-2015. 


\section{Calculation of DALY averted 2010-2015}

For each endemic country, knowing:

- the number of DALYs lost due to STH in 2010;

- the percentage of pre-SAC and SAC treated for 1 or more years with PC during 2010-2015;

and by applying the curves developed by Marocco et al. ${ }^{6}$ that predict the reduction in morbidity in a population treated at different times (1-10 years) for each of the STH parasites, we were able to estimate the number of DALYs averted by PC for each STH endemic country in 2015.

We summed up the national results of the number of DALYs averted by PC at global and regional levels for each STH species, and for the three STH species combined.

\section{Number of DALYs expected to be averted in $\mathbf{2 0 2 0}$}

We used a similar approach as described previously to calculate the number of DALYs that are expected to be averted in 2020 and we assumed that the $\mathrm{PC}$ will reach $75 \%$ coverage in each STH endemic country.

For each endemic country we calculated the yearly increase in coverage necessary to progressively reach the global target of $75 \%$ coverage of PC in 2020. We assumed a linear trend (e.g. if a country reached $50 \%$ PC coverage in 2015, we assumed a $5 \%$ increase every year to reach $75 \%$ coverage in 2020). If a country had already reached the global target in 2015, we assumed that it would maintain this level for the next 5 years.

Analogous to the methods used for the first objective, we used the estimated percentages of children treated consecutively for each time length between 2010 and 2020, along with the curves described by Marocco et al., ${ }^{6}$ to calculate the percentage of DALYS attributed to STH that are expected to be averted by $P C$.

Again, we summed up the national results of the number of DALYs expected to be averted by PC interventions at global and regional levels for each STH species and for the three STH species combined.

\section{Sensitivity analysis}

To address uncertainty in the reduction of moderate/high uncertainty, we also utilized the lower and upper values of the confidence interval of the curves defined by Marocco et al. ${ }^{6}$ to estimate DALYs averted in our study.

In addition to the variations on the reduction of infection of moderate/heavy intensity, we also estimated DALYs averted with the assumption that $75 \%$ coverage would be reached in each country 5 years later than anticipated (i.e. all countries reaching target coverage in in 2025 instead of 2020)

\section{Results}

\section{STH morbidity in the absence of PC interventions}

Globally, the total number of DALYs lost in 2010 (before the start of large-scale PC interventions) every year in all age groups due to STH infections was reported at 3.377 million, of which 0.399

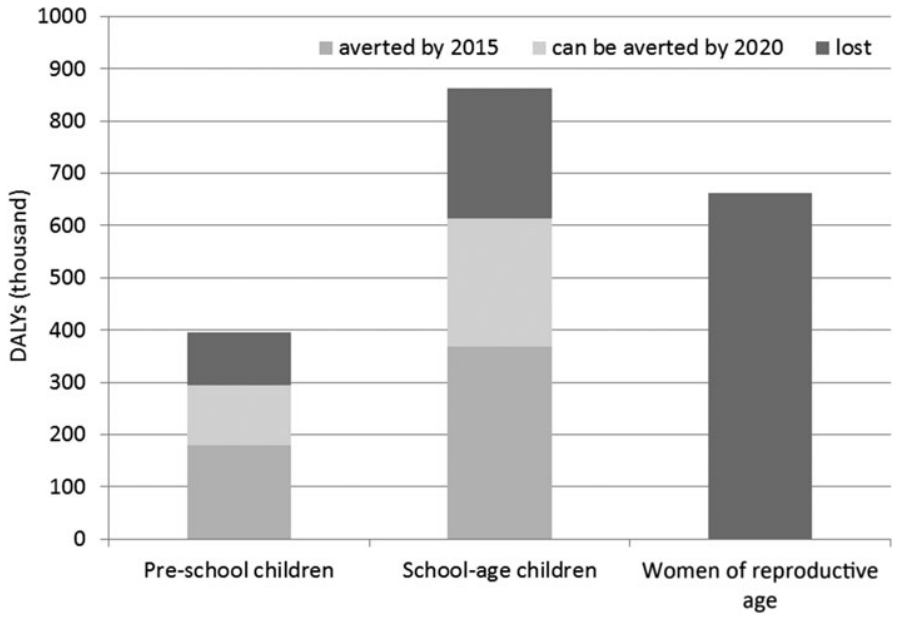

Figure 2. Number of DALYS lost because of STH before 2010, number of DALY averted in 2015 by the PC intervention and number if DALYs expected to be averted in 2020 in pre-SAC, SAC and WCBA.

million were lost in pre-SAC, 0.862 million in SAC and 0.622 million in WRA. In terms of STH species, 1.094 DALYs were lost to A. lumbricoides, 0.542 million were lost to T. trichiura and 1.739 million were lost to hookworms. ${ }^{8}$

Table 1 summarizes the morbidity in children attributed to the different STH species and the total averted morbidity (by STH species, age group and WHO region) in children in 2015.

In pre-SAC, most of the morbidity is due to $A$. lumbricoides (0.25 million DALYS), while in SAC the main morbidity is due to hookworms (0.45 million DALYs). The WHO African Region had the most DALYs due to STH (0.48 million) in the absence of PC interventions; $A$. lumbricoides is the parasite species responsible for the largest proportion of the disease burden.

Figure 2 shows the number of DALYs due to STH in the three groups at risk.

\section{Estimation of number of DALYs averted in 2015 by PC for STH conducted in 2010-2015}

In both subgroups of children, PC implemented during 2010-2015 is estimated to have averted more than 549000 DALYs in 2015, corresponding to $44 \%$ of the disease burden (in DALYs) present in 2010 before the global expansion of STH control programmes.

Approximately half of the DALYs from A. lumbricoides were averted. Most of the DALYs (283 000) were averted by control of A. lumbricoides and approximately 368000 of the 549000 DALYs averted were averted in SAC.

Figure 2 shows the number of DALYs averted by PC in the three groups at risk targeted in 2010-2015. Data on PC coverage among WCBA are not collected in detail and so, despite the fact that we know that a number of women of reproductive age is treated in the context of the global programme to eliminate lymphatic filariasis, we could not calculate the impact on DALYS in this group.

Table 1 summarizes the DALYs averted in 2015 due to the control of morbidity attributed to different STH species, in the different groups at risk and in the different $\mathrm{WHO}$ regions. 
In term of WHO regions, the South-East Asia Region has the most DALYs averted (220 000), corresponding to more than 57\% of the 2010 burden in the region.

\section{Estimation of the number of DALYs expected to be averted by PC for STH (2015-2020)}

An additional 360000 DALYs are expected to be averted by reaching the $75 \%$ global target for PC in 2020 (that focuses on pre-SAC and SAC), bringing the total reduction in DALYS due to STH to $73 \%$; this percentage is expected to approach $100 \%$ if, after reaching global target, PC is maintained for another 5 years.

Most of the additional reduction will be obtained by controlling hookworm infections (averting an additional 189000 DALYs), bringing the total reduction of the baseline burden of hookworm infections to nearly $70 \%$.

Table 1 summarizes also the DALYs expected to be averted due to the different STH species, in the different groups at risk and in the different WHO regions.

\section{Sensitivity analysis}

Using the lower and upper values of the CI for the three STH parasites we calculated that the number of DALYs averted in 2015 could fluctuate, for pre-SAC between 156000 and 216000 DALYs and for SAC between 319000 and 428000 DALYS.

In 2025 the fluctuation ranges between 202000 and 317000 DALY for pre-SAC and ranges between 432000 and 641000 DALYS for SAC.

If we contemplate reaching the $75 \%$ coverage target in 2025 instead of 2020, the DALYs averted in 2020 would be 238000 for PreSAC (instead of 295000 ) and 490000 for SAC (instead of $613000)$.

\section{Discussion}

We consider that a quantification of the benefits from the DALYS averted by PC conducted during 2010-2015 will allow the cost per DALY averted to be estimated and compared with that obtained by other interventions for other diseases.

\section{STH morbidity in the absence of PC interventions}

The 3.37 million DALYs attributed to STH in the absence of PC interventions are remarkable when compared with the DALYs attributed to other important diseases, such as Parkinson's disease (2.58 million) and acute hepatitis $B$ (3.5 million), which receive much more attention and investment for the control of their morbidity.

It is interesting that in pre-SAC most of the burden of STH is caused by A. lumbricoides (69\%) and in SAC most of the burden of STH is caused by hookworm infections, most likely due to the different age distributions of the two parasites. ${ }^{13}$

The fact that $T$. trichiura, the STH parasite more difficult to control with the anthelminthics currently used in $\mathrm{PC}^{14}$ is, in total, causing only $11 \%$ of the STH morbidity is noteworthy.

It is also interesting to compare the burdens in WHO's African and South-East Asia regions: in countries in the African
Region, fewer individuals are infected by $\mathrm{STH}$, but the morbidity is higher due to a higher prevalence of hookworm infections.

\section{STH morbidity averted during 2010-2015}

In our opinion, this is the first study to estimate the STH DALYS averted by PC interventions and, therefore, we do not have other estimations to compare with.

In the period 2010-2015, in which the donation of anthelminthics for STH was concretized and a steady increase of PC coverage was achieved, over $44 \%$ of morbidity in children was averted. If progress is sustained and the global target for PC coverage is reached as expected, the morbidity averted every year could be as much as $73 \%$ of the morbidity present before the intervention.

Despite these impressive results, we consider that our estimates of the number of DALYs averted by PC are conservative for two reasons:

- As depicted in Figure 1b, we did not account for treatments that were not continuous over time, meaning that in 68 out of 102 endemic countries a proportion of the PC treatments provided was not taken into consideration.

- Pre-SAC and SAC should be considered as transitory groups, so individuals who received PC treatment during childhood will continue to experience the benefit from the intervention in the subsequent phases of their life. A more detailed calculation would further add to the total number of DALYs averted.

It has not been possible to estimate the number of DALYS averted in WCBA because details on the number of individuals treated every year has been reported only from 2016. However, we expect PC coverage for this at-risk group to expand in the near future.

The WHO Global Health Estimates ${ }^{4}$ estimated a reduction of STH morbidity in children for 2015 (compared with 2010) in the order of $7 \%$. The present study estimates a morbidity reduction for STH in the order of $44 \%$ for the same interval (that is, six times higher). We consider the reason for this discrepancy to be the expansion of PC interventions during 2010-2015.

Of note is that the individuals in whom morbidity due to STH has been averted (or drastically reduced) frequently continue to live in highly contaminated environments with poor sanitation and are therefore exposed to re-infection. Despite the successes obtained until now by PC for STH, the intervention cannot be completely stopped for the moment even in areas where morbidity is now low. The frequency of PC can be progressively reduced, but PC cannot be abandoned until improvements in sanitation impede environmental contamination with human excreta. ${ }^{3}$ Even in our sensitivity analysis, using the lower CI bands from the Marocco et al. ${ }^{6}$ study, we find a significant number of DALYs averted from low/moderate intensity infections.

\section{Estimation of the number of DALYs expected to be averted by PC for STH (2015-2020)}

This estimation is conducted assuming that the efficacy of the drug will remain the same during 2015-2020. WHO is actively monitoring drug efficacy in countries that have been implementing PC for more than 5 years. For now there are no 
indications of reduced efficacy of albendazole or mebendazole on any of the STH parasites, but continuous monitoring of drug efficacy is necessary, ${ }^{14}$ in addition to measures to protect the efficacy of those drugs (e.g. targeting the intervention to groups at risk in order to maintain refugia where drug pressure is limited) we are testing drug combinations effective against STH to be deployed in case of resistance. ${ }^{15}$

In addition, we consider that the target of reaching $75 \%$ of children will be achieved in all endemic countries: coverage data received from countries for the 2016 drug requests for PC to be implemented in 2017 and 2018 show that the increase in global PC coverage is progressing better than expected. ${ }^{5}$ Nevertheless, we also estimated the number of DALYs averted should the coverage targets only be reached in 2025, showing that even in this scenario significant amount of DALYs would be averted.

We consider this estimation potentially useful to evaluate the cost utility of the investment made by several endemic countries on PC to control STH. One of the limitations is that we used the WHO Global Health Estimates; ${ }^{4}$ by using a different baseline evaluation of the DALYS caused by STH we would have obtained the same proportion, but a different total number of DALYs averted.

Reporting on DALY burden averted, in addition to reductions in infections and programme coverage, improves our understanding of the impact that these interventions have, and how they contribute to achieving the broader sustainable development goals. ${ }^{16}$

Author's contribution: MA and TW conceived the study; MA, MD, BM and JSA designed the study protocol; TW and MA carried out the analysis and interpretation of these data; MA and TW drafted the manuscript; BM, JSA and Fc critically revised the manuscript for intellectual content. All authors read and approved the final manuscript. MA is the guarantors of the paper.

\section{Acknowledgements: None.}

Funding: None.

Conflicts of interest: None declared.

Ethical approval: Not required (secondary analysis of already published data).

\section{References}

1 de Silva, NR, S Brooker, Hotez PJ et al. Soil-transmitted helminth infections: updating the global picture. Trends Parasitol 2003;19: 547-51.
2 Joseph S, Montresor A. Evaluation of the large-scale administration of drugs for the control of soil-transmitted helminthiasis. Part 1: Review of the evidence of morbidity. PloS Negl Trop Dis 2017; [in print]

3 Gabrielli A, Montresor A, Engels D et al. Preventive chemotherapy in human helminthiasis: theoretical and operational aspects. Trans R Soc Trop Med Hyg 2011;105:683-93.

4 WHO. Global Health Estimates 2000-2015: Disease burden by Cause, Age, Sex, by Country and by Region, 2000-2015. Geneva: World Health Organization; 2016. http://www.who.int/healthinfo/ global_burden_disease/estimates/en/index2.html (accessed 17 November 2017).

5 WHO. Eliminating soil transmitted helminthiasis as a public health problem in children. Progress report 2001-2010 and strategic plan2011-2020. Geneva: World Health Organization; 2012.

6 Marocco C, Bangert M, Joseph SA et al. Preventive chemotherapy in one year reduces by over $80 \%$ the number of individuals with soil transmitted helminthiases causing morbidity. Results from metaanalysis. Trans R Soc Trop Med Hyg 2017;111:12-17.

7 WHO. Helminth control in school age children. A guide for manager of control programmes. Geneva: World Health Organization; 2011.

$8 \mathrm{WHO} /$ Department of control of neglected tropical diseases. Schistosomiasis and soil-transmitted helminthiases: number of people treated in 2015. Wkly Epidemiol Rec 2016;91:585-600.

9 Yajima A, Mikhailov A, Mbabazi PS et al. Preventive chemotherapy and transmission control (PCT) databank: a tool for planning, implementation and monitoring of integrated preventive chemotherapy for control of neglected tropical diseases. Trans R Soc Trop Med Hyg. 2012;106:215-22.

10 WHO (2017) NTD databank available on line at http://www.who.int/ neglected_diseases/preventive_chemotherapy/databank/en/ (accessed 17 November 2017).

11 Pullan RL, Smith JL, Jasrasaria R et al. Global numbers of infection and disease burden of soil transmitted helminth infections in 2010. Parasit Vectors 2014;7:37.

12 Chan MS. The global burden of intestinal nematode infections-fifty years on. Parasitol Today 1997;13(11):438-43.

13 Renganathan E, Ercole E, Albonico $M$ et al. Evolution of operational research studies and development of a national control strategy against intestinal helminths in Pemba Island, 1988-92. Bull World Health Organ 1995;73(2):183-90.

14 WHO. Assessing the efficacy of anthelminthic drugs against schistosomiasis and soil transmitted helminthiasis. Geneva: World Health Organization; 2013.

15 Albonico M, Levecke B, LoVerde PT et al. Monitoring the efficacy of drugs for neglected tropical diseases controlled by preventive chemotherapy. J Glob Antimicrob Resist 2015;3:229-36.

16 Bangert M, Molyneux DH, Lindsay SW et al. The cross-cutting contribution of the end of neglected tropical diseases to the sustainable development goals. Infect Dis Poverty 2017;6(1):73. 